\title{
Sex and Mental Health Disorder Differences Among Military Service Members With Patellofemoral Syndrome
}

\author{
Daniel I. Rhon, DSc, Tanja C. Roy, PhD, Robert C. Oh, MD, MPH, FAAFP, and \\ Jodi L. Young, DPT
}

Background: Mental health disorders are associated with persistent knee pain, but the association between these conditions has had little investigation in the military. The purpose of this study was to identify rates of mental health disorders in patients with patellofemoral pain (PFP) and determine differences by sex and whether mental health copresence influences outcomes.

Methods: Eligible patients with a new PFP diagnosis were categorized according to sex and presence of mental health disorders. Outcomes included odds of mental health disorder before/after initial PFP diagnosis based on sex, and knee-related health care use between patients with/without mental health disorders.

Results: In 81,832 individuals with PFP (71.1\% men; mean age 33; 91.5\% active duty), copresence of any mental health disorders was common (18\% men; $28 \%$ women). Women had more depression and anxiety; men had more post-traumatic stress disorder and substance abuse disorders. Concurrent mental health disorders after initial PFP diagnosis resulted in higher medical costs and odds of a recurrence (OR 1.24; 95\% CI 1.20, 1.28; $P<.001)$.

Conclusion: Mental health disorders are common in military service members seeking care for patellofemoral pain. Differences in prevalence vary by sex, and presence of mental health disorders adversely affected long-term health care outcomes. (J Am Board Fam Med 2021;34:328-337.)

Keywords: Connective Tissue, Gender Differences, Health Services Research, Mental Health, Military Medicine, Musculoskeletal Pain, Patellofemoral Pain Syndrome, Veterans Health

\section{Introduction}

Musculoskeletal disorders are the leading cause of limited duty days in the US military, with the lower extremity being most commonly affected. ${ }^{1}$ Patellofemoral pain (PFP) is the single most common musculoskeletal complaint of the lower extremity in this same population, with a point prevalence as high

This article was externally peer reviewed.

Submitted 30 June 2020; revised 19 December 2020; accepted 21 December 2020.

From the Primary Care Musculoskeletal Research Center, Brooke Army Medical Center, JBSA Fort Sam Houston, TX (DIR, JLY); Department of Rehabilitation Medicine, Uniformed Services University of Health Sciences, Bethesda, MD (DIR); U.S. Army Research Institute of Environmental Medicine, Natick, MA (DIR, TCR): Department of Family Medicine, Madigan Army Medical Center, Tacoma, WA (RCO): Doctor of Science in Physical Therapy Program, Bellin College, Green Bay, WI (DIR, JLY).

Funding: No funding was received for this project.

Conflict of interest: None declared.

Disclaimer: The view(s) expressed herein are those of the author(s) and do not reflect the official policy or position of as $13.5 \% .^{2}$ Recently, psychological factors such as depression, anxiety, catastrophizing, and fear of movement have been reported at high proportion in patients with PFP, ${ }^{3,4}$ and these psychological factors have been associated with high levels of disability and worse levels of function. ${ }^{4}$ This is relevant because service members also suffer from higher rates of mental health disorders compared with civilian populations, especially in those with deployment history, ${ }^{5,6}$ which could also hinder recovery from highly prevalent conditions such as PFP-related disorders.

Brooke Army Medical Center, the Uniformed Services University of Health Sciences, the U.S. Army Research Institute of Environmental Medicine, the U.S. Army Office of the Surgeon General, the Department of the Army, the Defense Health Agency, the Department of Defense, or the U.S. government.

Corresponding author: Daniel I. Rhon, DSc, Primary Care Musculoskeletal Research, Department of Rehabilitation Medicine, Brooke Army Medical Center, 3551 Roger Brooke Drive, JBSA Fort Sam Houston, TX 78234 (E-mail: daniel.i.rhon.ctr@mail.mil). 
There is a known disparity in rates between men and women for both PFP and mental health disorders. Female military cadets are 2.23 times more likely to develop PFP than males, ${ }^{7}$ and military females in the entire US military had an incidence rate ratio for PFP of $1.50(95 \%$ CI $1.47,1.54)$ compared with males from 2006 to $2012 .{ }^{8}$ While females make up approximately $15 \%$ of the US active duty military force, ${ }^{9}$ they were responsible for $31.8 \%$ of all of the incident cases of PFP in the Military Health System (MHS) over a 2year period. ${ }^{10}$ Females are twice as likely to be diagnosed with post-traumatic stress disorder (PTSD), ${ }^{11}$ anxiety, ${ }^{12}$ and depression. ${ }^{13}$ In addition, depression is a risk factor for knee pain, ${ }^{14}$ and psychological health is associated with pain and physical function in patients with chronic knee pain. ${ }^{15,16}$ While relationships between mental health and physical health have been previously proposed, ${ }^{17}$ very little has been done to understand the impact or sequelae of these differences. Because mental health disorders are known to negatively impact outcomes for PFP, and pain processing varies by sex, ${ }^{18}$ this relationship requires further investigation in military settings. No work to date has focused on mental health profiles in beneficiaries of the MHS with PFP. The purpose of this study was to assess the differences in mental health disorders by sex in MHS beneficiaries seeking medical care for PFP and their influence on health care outcomes. This is important because not only can physical approaches to rehabilitation of PFP vary by sex, ${ }^{19}$ treatments for mental health disorders may also be uniquely tailored based on sex. ${ }^{20,21}$ The findings will better inform women's health programs and provide a clearer understanding of the intersectionality underpinning sexual dimorphisms in pain and symptoms associated with mental health disorders. The hypothesis was that patients with a prior history of a mental health disorder, or those who develop a mental health disorder after the initial knee pain medical visit, would have worse outcomes (higher costs, greater health care use, and higher numbers of recurrence) compared with patients without a mental health disorder diagnosis. Rates of mental health disorders were also expected to be higher in women, as previously reported in populations not specific to those with PFP.

\section{Methods}

\section{Study Design}

This was a retrospective population-level cohort study using electronic medical records and claims data. We used the REporting of studies Conducted using Observational Routinely-collected health Data $(\mathrm{RECORD})^{22}$ extension of Strengthening the Reporting of Observational Studies in Epidemiology to guide the reporting of these findings.

\section{Setting}

Military and civilian clinics worldwide within the MHS were included. Ethics and regulatory approval were provided by the Institutional Review Board of the Army Regional Health Command Central in San Antonio, Texas.

\section{Participants}

Participants were TRICARE beneficiaries (the sole government-backed insurance program for military beneficiaries) seeking medical care for knee pain in military or civilian clinics between January 1, 2010 and December 31, 2011 and rendered a diagnosis of PFP. The International Classification of Diseases, Ninth Revision (ICD-9) code 717.7, "chondromalacia patellae," was used identify the reason for the medical visit and target diagnosis. ${ }^{23,24} \mathrm{We}$ excluded anyone with any knee-related care in the 1 year before to ensure it was an initial visit for knee pain, as well as anyone with a cancer diagnosis (due to differences in management of cancer-related pain) any time before or after the initial knee injury. Patients who were not eligible beneficiaries for the entire 12 months before and 24 months after the initial diagnosis (surveillance period of January 1, 2009 through December 13, 2013) were also excluded. To improve the specificity of the patellofemoral diagnosis, individuals with a concomitant diagnosis of patellar tendinopathy, iliotibial band friction syndrome, cruciate ligament or meniscus injuries, knee joint sprain, knee osteoarthritis, knee fracture, or the presence of a surgical knee procedure, all within 6 months after diagnosis, were also excluded. The cohort was derived initially to align best with individuals on active duty, and therefore only included adults between the ages of 17 (17 if they were an emancipated minor on active duty) to 50 years (Figure 1). Retired veterans in this age range were also included.

\section{Data Source}

Electronic medical records and claims data of participants were sourced from the US Military Health System Data Repository (MDR). The MDR is a centralized data repository that captures Defense 
Figure 1. Flow of cohort identification.

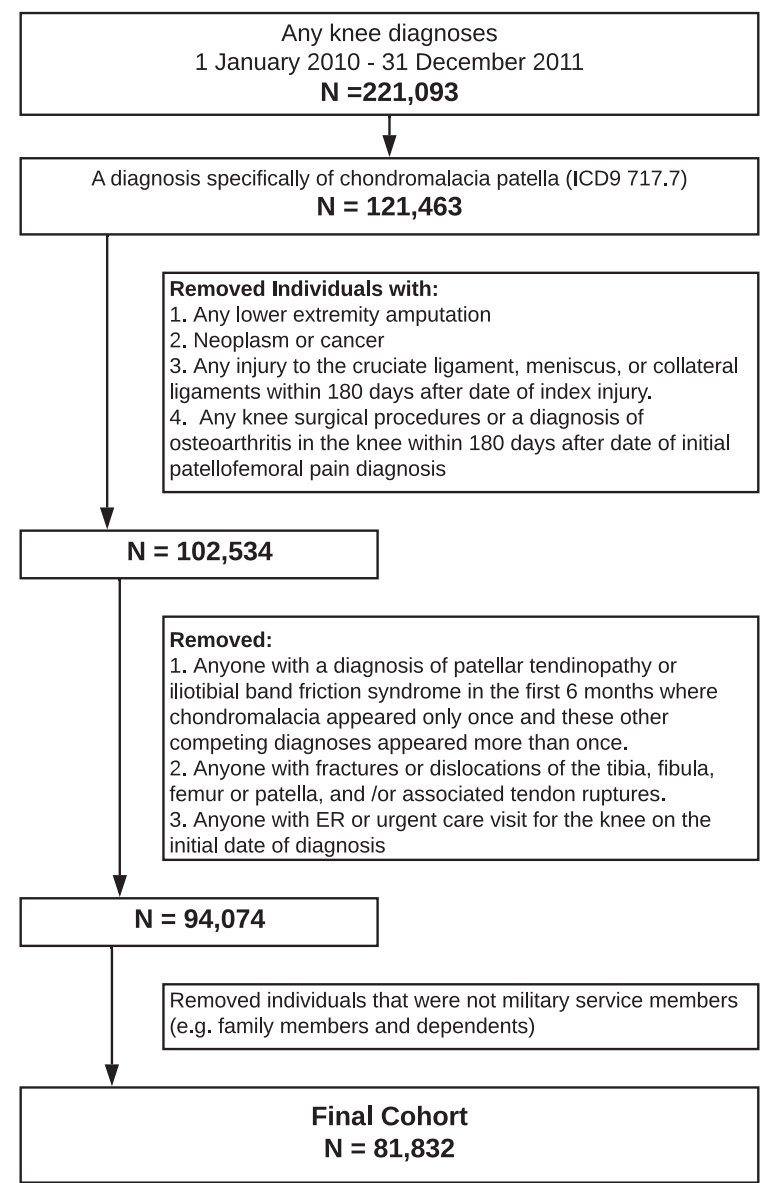

Health Agency (DHA) corporate health care data worldwide. The variables include person-level data for all outpatient and inpatient medical visits, both in military and civilian hospitals, encompassing all medical and surgical procedures and all medication prescriptions for all beneficiaries. The person-level data were provided without identifiers to the investigators with a sum of each care variable for each patient.

\section{Variables}

\section{Groups}

The mental health diagnoses of interest included depression, anxiety, and PTSD with the use of ICD codes as previously described. ${ }^{5}$ To increase sensitivity, we also included individuals that were rendered a mental health disorder diagnosis of any kind (ICD-9 codes 290.xx through 316.xx). ${ }^{5}$ Patients who had a diagnosis for a substance use disorder were also identified. Individuals with at least 2 medical visits for the diagnosis of interest were classified as having the disorder. This criterion is used by the Armed Forces Health Surveillance Branch (AFHSB), the central epidemiologic resource for the US Armed Forces, for their routine surveillance and reporting. ${ }^{5}$ Groups were created based on the presence of the relevant diagnosis both before and after the initial PFP visit. For sensitivity, we also created a group with a new diagnosis after the initial PFP visit (diagnosis present after, but not before).

\section{Outcomes}

Our outcomes were total knee-related medical visits, total cost of knee-related care, recurrence of knee pain (new episodes), and use of opioids. For the entire 2-year period after the original medical visit for PFP, we identified all visits for any kneerelated care and the total costs of that care. This included any knee-related procedures (injections, radiology tests, or surgical procedures) and consultations in any outpatient clinical setting, either military or civilian. We identified any new knee-related care following a minimum of 60 days without any knee care as a new episode (eg, recurrence), which is the criteria used by the Army Public Health Center for determination of a new injury. ${ }^{25}$ Opioid prescription fills were identified from the Pharmacy Data Transaction Service (PDTS) within the MDR based on American Hospital Formulary Service (AHFS) classification codes of 280808 and 280812.

\section{Data Analyses}

Demographic information was reported by sex and presence of each mental health disorder before the initial knee pain visit. Chi squared analysis was used to calculate odds ratios with $95 \%$ confidence intervals for sex and mental health disorder presence as well as for dichotomous outcome variables (recurrent episodes and opioid prescription fill occurrence). Absolute and relative risk ratios were calculated for differences in mental health condition prevalence by sex. Continuous outcomes were calculated using a generalized linear model with negative binomial regression for count data (knee-related visits) and with $\gamma \log$ transformation for knee-related costs for comparisons between groups (with and without mental health disorder). These adjustments were made due to the presence of skewed data, which is typical with health care use. ${ }^{26}$

\section{Missing Data}

The MDR has a robust system for accounting for missing data. Various data sources are fed in 
"RAW" form into the database where all missing data are continuously validated for up to 90 days before becoming "FINAL" validated data. We did not pull MDR data within 90 days of the last follow-up date to ensure use of fully validated data. Because it is a closed single-payer health system, if a medical event was missing, it was assumed to not have happened.

\section{Results}

Of the 81,832 unique individuals included in the final cohort (Figure 1), 58,203 (71.1\%) were men (mean age $33.7 ; 89.4 \%$ on active duty) and 23,629 (28.9\%) were women (mean age 32.4; 96.6\% active duty). Baseline demographics are shown in Table 1.

\section{Differences between Sexes}

Overall, there was a large proportional increase in all cases of mental health disorders from before or after the initial PFP diagnosis, in both male and female service members. For men, there was an increase in any mental health disorders (136.9\%), depression (149.6\%), PTSD (173.8\%), and anxiety $(178.3 \%)$. For women, there was an increase in any mental health disorders (77.3\%), depression (81.1\%), PTSD (118.2\%), and anxiety (138.8\%). Every individual with depression, PTSD, anxiety, and substance use disorders had at least 1 other concurrent mental health diagnosis, rather than a single diagnosis alone (Figure 2).

Mental health disorders were found at significantly higher rates in women regardless of whether the diagnosis occurred before or after the initial visit for knee pain $(\mathrm{OR}=2.05,95 \%$ CI 1.96-2.15 before; $\mathrm{OR}=1.6195 \%$ CI 1.56-1.67 after; see Figure 3 for specific disorders). The exception was PTSD and substance use disorders, in which female sex was protective before and after the initial knee pain diagnosis (Figure 3). The absolute risk reduction was not as stark, however, ranging from a $4.9 \%$ to $8.5 \%$ risk reduction in men compared with women for any mental health disorder, anxiety, and depression, and a $1.3 \%$ and $2.6 \%$ absolute risk increase in substance use and PTSD, respectively, in men compared with women (Table 2). When looking specifically at retired veterans, only depression (OR 1.71, 95\% CI 1.31-2.24 $P<.001$ ) and anxiety (OR 1.56, 95\% CI 1.16-2.11; $P=.003$ ) after the initial PFP diagnosis were significantly higher in women. When considering only new cases of depression, not present rendered before the initial PFP diagnosis, the difference in depression by sex was no longer significant (OR 1.33, 95\% CI 0.93 $1.90 ; P=.120)$.

\section{Health Care Use}

Mental health disorders were significantly associated with the amount of knee-related health care use outcomes, but the association was stronger and more often significant when the mental health disorder diagnosis was present after the initial knee pain episode compared with before (Table 3 ). The exception was the diagnosis of substance use disorders; only their presence before the initial knee pain visit resulted in significantly greater 2-year knee-related medical costs and visits, but the presence after did not influence outcomes. Mean medical costs ranged from $\$ 200$ to $\$ 456$, and mean medical visits ranged from 1 to 2.2 more per individual with $\mathrm{PFP}$ who also had a mental health diagnosis compared with those without the mental health diagnosis (Table 3). The odds of having at least 1 or more additional episodes of knee pain in the 2-year follow-up period were significantly higher for those with a mental health disorder, either before or after (OR 1.24, 95\% CI 1.20-1.28; $P<.001)$. Patients with PFP and a mental health disorder were also significantly more likely to receive at least 1 opioid prescription fill during the 2-year follow-up (OR 1.37, 95\% CI 1.29-1.45; $P<.001)$.

\section{Discussion}

The findings from this cohort suggest patients who seek medical care for PFP with a concurrent mental health disorder are also likely to have worse kneerelated outcomes that include persistent pain (more knee pain episodes), a greater number of total medical visits, and higher overall knee-related medical costs. In addition, female service members seeking medical care for PFP, in particular those still on active duty, are much more likely to also receive a concurrent diagnosis of depression or anxiety. Male service members with similar knee pain are more likely to have a concurrent diagnosis of PTSD or a substance use disorder. This relationship suggests that awareness of a history of mental health disorders or considering a comorbid diagnosis for these disorders when patients present with musculoskeletal knee pain could potentially help set the 


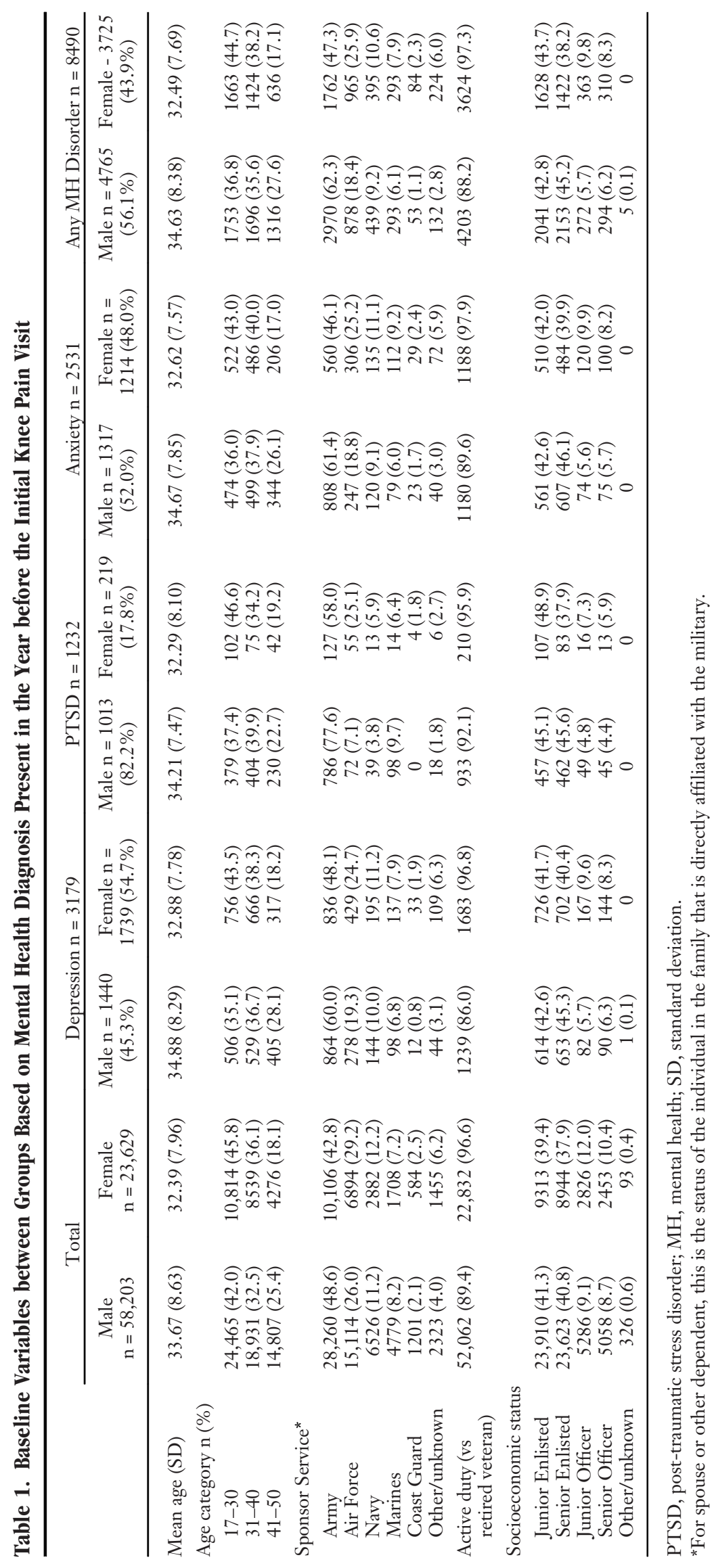


Figure 2. Concurrent presentation of mental health disorders before and after the initial patellofemoral knee pain diagnosis. PTSD, post-traumatic stress disorder.

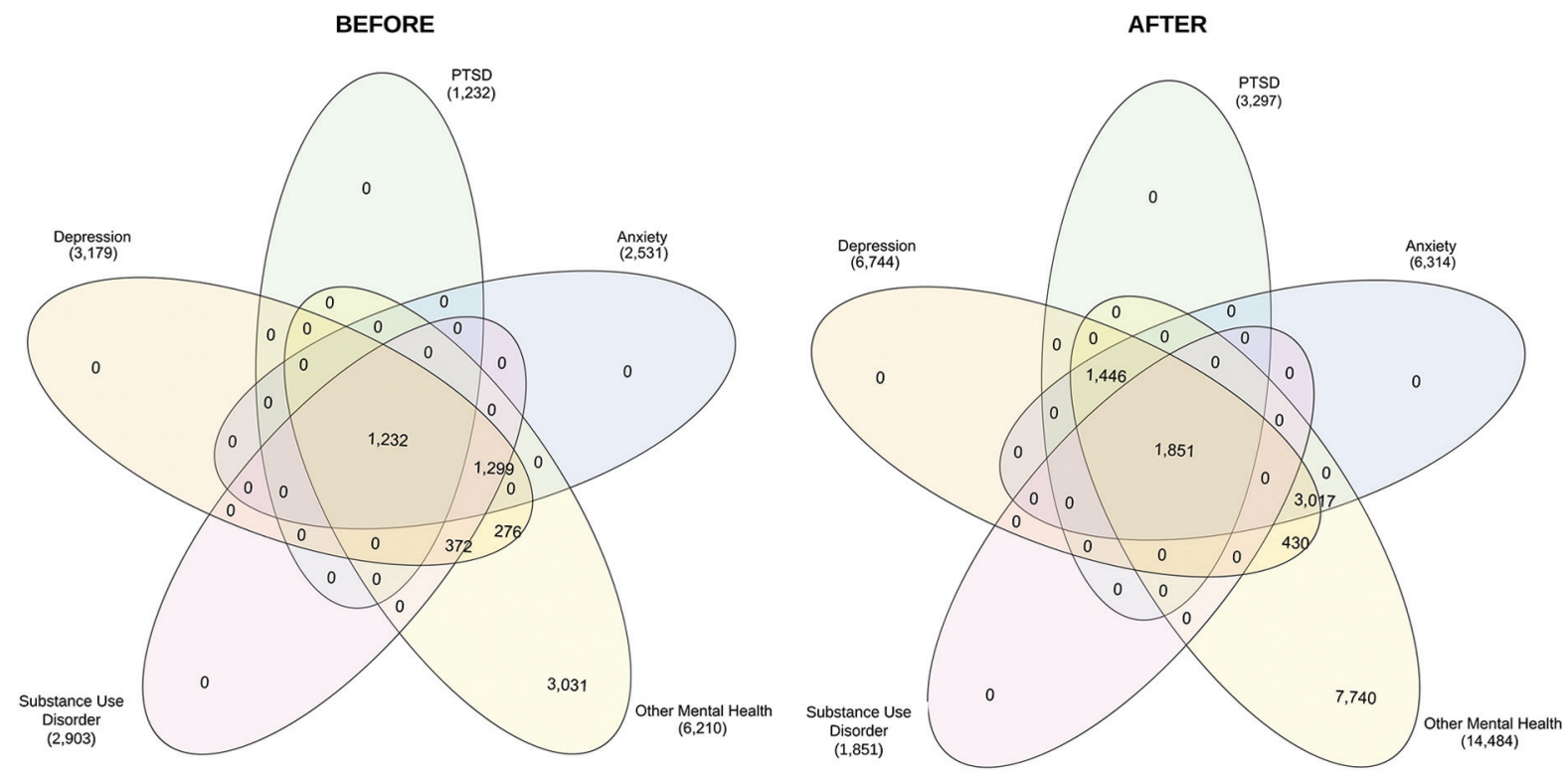

Figure 3. Odds of mental health disorder presence based on sex. Odds are shown for females, with men as the reference standard (eg, women had 2.34 times the odds for a depression diagnosis before and 1.98 times the odds for a depression diagnosis after the initial patellofemoral pain diagnosis). PTSD, post-traumatic stress disorder.

BEFORE

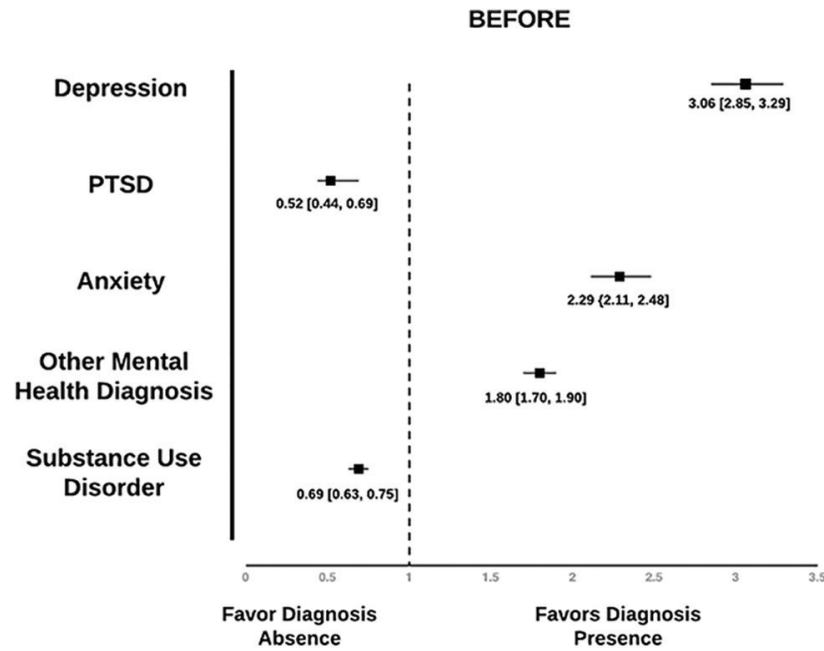

foundation for improving long-term care and outcomes in patients with PFP.

A recent systematic review identified 4 psychological constructs associated with PFP: mental health, cognitive factors, behavioral factors, and other factors. ${ }^{4}$ The mental health factors specifically included anxiety, depression, and general mental health, which correlated with pain and

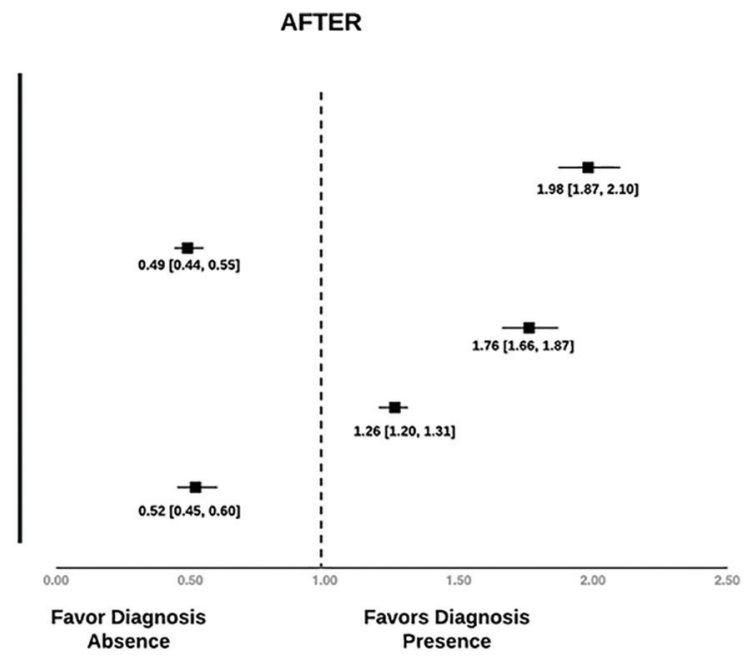

physical function. ${ }^{4}$ Some argue that symptoms of chronic PFP better align with evidence of nociplastic pain (altered nociception despite actual or threatened tissue $)^{27}$ than traditional nociceptive models, ${ }^{3}$ the former of which lends itself to greater central sensitization and widespread reduced pain thresholds. This type of pain also has a stronger association with maladaptive 
Table 2. Relative Risk of Behavioral and Mental Health Diagnosis After Initial Knee Pain Visit With Men As the Reference Standard

\begin{tabular}{lccc}
\hline & $\begin{array}{c}\text { Risk Ratio } \\
(95 \% \mathrm{CI})\end{array}$ & $\begin{array}{c}\text { Relative Risk Reduction } \\
(95 \% \mathrm{CI})\end{array}$ & $\begin{array}{c}\text { Absolute Risk Reduction } \\
(95 \% \mathrm{CI})\end{array}$ \\
\hline PTSD & $0.46(0.42,0.51)$ & $53.6 \%(49.1,57.7)$ & $2.6 \%(2.3,2.8)$ \\
Depression & $2.16(2.06,2.26)$ & $-116.9 \%(-125.9,-106.3)$ & $-7.2 \%(-7.6,-6.7)$ \\
Anxiety & $1.78(1.70,1.87)$ & $-78.0 \%(-86.7,69.8)$ & $-4.9 \%(-5.4,-4.5)$ \\
Any mental health disorder & $1.44(1.40,1.48)$ & $-44.0 \%(-47.9,-40.3)$ & $-8.5 \%(-9.2,-7.9)$ \\
Substance use disorder & $0.51(0.45,0.57)$ & $49.5 \%(43.0,55.2)$ & $1.3 \%(1.1,1.5)$ \\
\hline
\end{tabular}

PTSD, post-traumatic stress disorder.

Values represent the risk for women in comparison to men. Negative numbers for relative or absolute risk indicate an increase in risk versus a reduction (ie, females had a negative relative risk reduction [-116.9\%] for depression, indicating their risk increased).

Table 3. Health Care Use Outcomes Based on the Presence of Mental Health Disorders

\begin{tabular}{|c|c|c|c|c|}
\hline & $\begin{array}{l}\text { Total 2-Year Knee- } \\
\text { Related Medical Costs } \\
\text { Mean USD (95\% CI) }\end{array}$ & $\begin{array}{l}\text { Total Knee- } \\
\text { Related Care Visits } \\
\text { Mean }(95 \% \text { CI })\end{array}$ & $\begin{array}{c}\text { Two or More Episodes of } \\
\text { Knee Pain (Recurrence) } \\
\text { Odds Ratio (95\% CI) }\end{array}$ & $\begin{array}{c}\text { At Least One Opioid } \\
\text { Prescription Fill Odds } \\
\text { Ratio }(95 \% \mathrm{CI})\end{array}$ \\
\hline $\begin{array}{l}\text { No MH disorder } \\
\text { diagnoses before }\end{array}$ & $1073(1057,1089)$ & $4.87(4.82,4.93)$ & & \\
\hline No MH diagnoses after & $1031(1014,1048)$ & $4.67(4.62,4.73)$ & & \\
\hline $\begin{array}{l}\text { No MH diagnoses } \\
\text { before or after }\end{array}$ & $1037(1019,1054)$ & $4.70(4.64,4.76)$ & & \\
\hline \multicolumn{5}{|c|}{ MH diagnosis present before diagnosis of patellofemoral pain } \\
\hline Depression & $1076(1007,1149)$ & $5.05(4.78,5.33)$ & $0.98(0.91,1.06)$ & $1.27(1.11,1.44)^{*}$ \\
\hline PTSD & $1175(1073,1286)$ & $5.27(4.85,5.71)$ & $1.30(1.16,1.46)^{*}$ & $1.26(1.02,1.54)^{*}$ \\
\hline Anxiety & $1065(997,1138)$ & $5.14(4.84,5.45)$ & $1.08(0.995,1.17)^{*}$ & $1.35(1.17,1.56)^{*}$ \\
\hline Any $\mathrm{MH}$ disorder & $1087(1046,1129)$ & $5.06(4.90,5.22)^{*}$ & $1.11(1.07,1.17)^{*}$ & $1.27(1.16,1.38)^{*}$ \\
\hline Substance use disorder & $1158(1081,1241)^{*}$ & $5.18(4.89,5.49)^{*}$ & $1.10(1.02,1.19)^{*}$ & $1.19(1.03,1.37)^{*}$ \\
\hline \multicolumn{5}{|c|}{$\mathrm{MH}$ diagnosis present after diagnosis of patellofemoral pain } \\
\hline Depression & $1244(1187,1303)^{*}$ & $5.73(5.53,5.93)^{*}$ & $1.40(1.33,1.48)^{*}$ & $1.43(1.31,1.56)^{*}$ \\
\hline PTSD & $1487(1400,1579)^{*}$ & $6.60(6.28,6.95)^{*}$ & $1.89(1.76,2.02)^{*}$ & $1.56(1.38,1.75)^{*}$ \\
\hline Anxiety & $1263(1204,1324)^{*}$ & $5.92(5.70,6.14)^{*}$ & $1.33(1.28,1.41)^{*}$ & $1.46(1.34,1.60)^{*}$ \\
\hline Any $\mathrm{MH}$ disorder & $1231(1197,1266)^{*}$ & $5.67(5.55,5.80)^{*}$ & $1.41(1.36,1.45)^{*}$ & $1.42(1.33,1.51)^{*}$ \\
\hline Substance use disorder & $1120(1027,1221)$ & $5.28(4.91,5.67)$ & $1.27(1.16,1.39)^{*}$ & $1.21(1.02,1.44)^{*}$ \\
\hline \multicolumn{5}{|c|}{ New MH diagnosis present after diagnosis of patellofemoral pain } \\
\hline Depression & $1275(1206,1348)^{*}$ & $5.86(5.63,6.10)^{*}$ & $1.43(1.35,1.52)^{*}$ & $1.45(1.30,1.60)^{*}$ \\
\hline PTSD & $1552(1443,1669)^{*}$ & $6.87(6.47,7.29)^{*}$ & $2.03(1.87,2.20)^{*}$ & $1.61(1.40,1.84)^{*}$ \\
\hline Anxiety & $1301(1230,1376)^{*}$ & $6.03(5.78,6.29)^{*}$ & $1.39(1.31,1.48)^{*}$ & $1.49(1.35,1.65)^{*}$ \\
\hline Any $\mathrm{MH}$ disorder & $1264(1220,1310)^{*}$ & $5.80(5.64,5.97)^{*}$ & $1.42(1.37,1.48)^{*}$ & $1.37(1.27,1.47)^{*}$ \\
\hline Substance use disorder & $1099(994,1215)$ & $5.23(4.81,5.68)^{*}$ & $1.38(1.24,1.54)^{*}$ & $1.26(1.03,1.54)^{*}$ \\
\hline
\end{tabular}

MH, mental health; PTSD, post-traumatic stress disorder; USD, US dollars; OR, odds ratio; CI, confidence interval.

${ }^{*}$ Value is significant at $P<.05$. Compared to NO mental health disorder either before or after initial patellofemoral pain diagnosis, the odds of having more than 1 knee pain episode were $\mathrm{OR}=1.24$ (95\% CI 1.20, 1.28) and for filling at least 1 opioid prescription were $\mathrm{OR}=1.37(95 \%$ CI $1.29,1.45)$.

psychological profiles (pain catastrophizing, kinesiophobia, lower self-efficacy) and mental health disorders such as depression. ${ }^{28}$ In a recent trial of 150 patients with PFP, psychological factors explained $40 \%$ of the variance in pain and physical function. ${ }^{3}$ It is important to clarify that this only represents a subset of patients with PFP, more likely the ones with chronic symptoms.

The findings we report in this cohort from within the MHS that connect mental health and knee pain corroborate those reported in other populations. However, no other study has reported 
differences in mental health disorders by sex within this specific patient population and how those mental health disorders could influence other relevant health-seeking outcomes. These findings are novel and provide a deeper understanding of the relationship between PFP, mental health disorders, and health care use.

While depression and psychological health have been identified as risk factors for knee pain, ${ }^{14,15}$ their presence before the initial knee pain visit was not a strong predictor of poor outcomes in this cohort. The association was much stronger after the initial knee pain visit, and it is not possible to know if the mental health disorder exacerbated the physical knee symptoms or vice versa. In part, this could be attributed to the timing of the diagnosis, which does not likely represent the actual beginning of the mental health symptoms. The stigma associated with seeking care for mental health can often delay the diagnosis, and the symptoms may have been present before the physical patellofemoral knee pain or even exacerbated by it. ${ }^{29}$ Pressure to return to full military duty can drive increased anxiety and catastrophizing, which leads to longer recovery periods and poorer long-term function. ${ }^{30-32}$ PTSD is associated with changes in cognitive function, ${ }^{33}$ and cognition is known to be a key component of pain modulation. ${ }^{34}$ This may help explain why patients with PTSD were more likely to have a greater number of recurrences of knee pain and had the highest knee-related medical costs out of all of the groups. The difference in presentation between male and female service members provides an opportunity for implementation of individualized and whole-person approaches to the management of musculoskeletal knee pain. The volume of patients presenting to primary care clinics with a primary complaint of knee pain is much higher than that presenting with primary mental health symptoms. In 2019, overuse musculoskeletal injuries like PFP were responsible for more than $70 \%$ of all limited duty days across the entire US Army, while behavioral and mental health conditions accounted for only $6 \%$ during the same time period. ${ }^{1}$ Proper screening for depression, anxiety, PTSD, and catastrophizing can help predict outcomes and lead to appropriate whole-person care strategies at earlier points in the treatment pathway. Pain catastrophizing, for example, predicts greater long-term medical visits and costs for other musculoskeletal disorders in military populations ${ }^{35}$ and may be of value as a screening tool in patients with PFP.
Catastrophizing, a tendency to magnify the threat level (rumination, magnification, helplessness), ${ }^{36}$ is also one of the stronger moderators between pain intensity and opioid prescription, ${ }^{37}$ which could explain the higher rates of opioid use in those with a concurrent mental health disorder. Attention should be paid to how these presentations may differ between male and female service members, especially considering the sexual dimorphisms associated with pain control, ${ }^{38}$ the disparity between sexes for chronic pain medical care received by veterans, ${ }^{39}$ and the higher likelihood of ostracism faced by military females, which may also influence psychological health and pain perception. ${ }^{40}$

\section{Strengths and Limitations}

The main strength of this study is the large sample size, representing the entire population rather than just a sample, as well as the 2-year follow-up period and 1-year lookback period. Another strength is that the MHS is a closed single-payer system, where there are no copays or limits to medical care access by active-duty service members, which means it was very unlikely care occurred elsewhere and was not captured. There were also several limitations to this study. The diagnostic codes for mental health disorders are only as accurate as the providers using them, and there could be potential variability in diagnostic accuracy among providers and even geographically. However, these are the same codes and processes used by the AFHSB to identify these mental health disorders in their epidemiologic reports. For the same reason, there is a chance that the code for "chondromalacia patellae" did not capture all instances of PFP, and some may have been missed or coded incorrectly. We attempted to mitigate this issue with our screening process by excluding cases that had other concurrent knee diagnoses (eg, cruciate ligament, meniscus injury, etc.). While other generic knee pain codes (ie, 719.46) can be used to code PFP, they also include many other non-PFP disorders. Including them would have increased sensitivity but sacrificed specificity of the results for PFP, which we felt was most important for this particular question. Finally, to assess long-term outcomes in this sample, we only included participants with at least 2 years of follow-up. These findings may be different when including every service member, regardless of available follow-up period. However, inclusion of everyone would have limited the extent of the 
outcomes we collected. While the relationship between mental health disorders and outcomes is strong, conclusions about causation cannot be made from this study. Finally, these data reflect care patterns from the MHS that may no longer be current, needing further validation. While opioid use and substance use disorders in the MHS in general have been decreasing, ${ }^{41}$ annual rates of musculoskeletal injuries to include PFP and mental health disorders have been increasing. ${ }^{1,5}$ This issue likely still carries relevance to current practice. These findings also provide an excellent rationale for further study of sex-specific differences in mental health disorders for patients with PFP, to determine if improvement with one can result in improvement in the other. Prospective trials are needed to further understand the nature of this relationship.

\section{Conclusion}

Approximately $19 \%$ of male and $28 \%$ of female military service members seeking medical care for PFP had a mental health disorder in the 2 years after the initial PFP visit. The presence of a mental health disorder before and after the initial PFP visit was associated with greater medical visits, higher costs, greater likelihood of knee pain recurrence, and greater likelihood of filling an opioid prescription in the following 2 years compared with individuals without a mental health diagnosis. Female service members, especially those on active duty (compared with retired veterans), were more likely to have depression, anxiety, or any mental health disorder in general. Male service members were more likely to have a PTSD or substance use disorder diagnosis. Clinicians should consider these relationships and their implications when managing patients with PFP.

To see this article online, please go to: http://jabfm.org/content/ 34/2/328.full.

\section{References}

1. Molloy JM, Pendergrass TL, Lee IE, Chervak MC, Hauret KG, Rhon DI. Musculoskeletal injuries and United States Army readiness part i: overview of injuries and their strategic impact. Mil Med 2020;185:e1461-e1471.

2. Smith BE, Selfe J, Thacker D, et al. Incidence and prevalence of patellofemoral pain: a systematic review and meta-analysis. PLoS One 2018;13: e0190892.
3. Maclachlan LR, Collins NJ, Hodges PW, Vicenzino B. Psychological and pain profiles in persons with patellofemoral pain as the primary symptom. Eur J Pain 2020;24:1182-96.

4. Maclachlan LR, Collins NJ, Matthews MLG, Hodges PW, Vicenzino B. The psychological features of patellofemoral pain: a systematic review. $\mathrm{Br}$ J Sports Med 2017;51:732-42.

5. Stahlman S, Oetting AA. Mental health disorders and mental health problems, active component, U.S. Armed Forces, 2007-2016. MSMR 2018;25:2-11.

6. Morgan JK, Levin-Rector A, Van Dorn RA, et al. Trends in mental health outcomes and combat exposure among US Marines returning from Iraq, Afghanistan or other deployments, 2004-13. J Public Health (Oxf) 2019;41:313-20.

7. Boling M, Padua D, Marshall S, Guskiewicz K, Pyne S, Beutler A. Gender differences in the incidence and prevalence of patellofemoral pain syndrome. Scand J Med Sci Sports 2010;20: 725-30.

8. Kusnezov N, Watts N, Belmont PJ, Jr, Orr JD, Waterman B. Incidence and risk factors for chronic anterior knee pain. J Knee Surg 2016;29:248-53.

9. Bean-Mayberry B, Huang C, Batuman F, et al. Systematic review of women veterans health research 2004-2008. Department of Veterans Affairs (US); 2010.

10. Garcia AN, Cook CE, Rhon DI. Adherence to stepped care for management of musculoskeletal knee pain leads to lower health care utilization, costs, and recurrence. Am J Med 2020; S0002-9343(20) 30778-6.

11. Christiansen DM, Hansen M. Accounting for sex differences in PTSD: a multi-variable mediation model. Eur J Psychotraumatol 2015;6:26068.

12. Altemus M, Sarvaiya N, Neill Epperson C. Sex differences in anxiety and depression clinical perspectives. Front Neuroendocrinol 2014;35:320-30.

13. Labaka A, Goñi-Balentziaga O, Lebeña A, PérezTejada J. Biological sex differences in depression: a systematic review. Biol Res Nurs 2018;20:383-92.

14. Phyomaung PP, Dubowitz J, Cicuttini FM, et al. Are depression, anxiety and poor mental health risk factors for knee pain? A systematic review. BMC Musculoskelet Disord 2014;15:10.

15. Iijima H, Aoyama T, Fukutani N, et al. Psychological health is associated with knee pain and physical function in patients with knee osteoarthritis: an exploratory cross-sectional study. BMC Psychol 2018;6:19.

16. Urquhart DM, Phyomaung PP, Dubowitz J, et al. Are cognitive and behavioural factors associated with knee pain? A systematic review. Semin Arthritis Rheum 2015;44:445-55.

17. Ohrnberger J, Fichera E, Sutton M. The relationship between physical and mental health: a mediation analysis. Soc Sci Med 2017;195:42-9. 
18. Mogil JS. Qualitative sex differences in pain processing: emerging evidence of a biased literature. Nat Rev Neurosci 2020;21:353-65.

19. Zawadka M, Smolka J, Skublewska-Paszkowska M, et al. Sex-dependent differences in single-leg squat kinematics and their relationship to squat depth in physically active individuals. Sci Rep 2020;10:19601.

20. Sramek JJ, Murphy MF, Cutler NR. Sex differences in the psychopharmacological treatment of depression. Dialogues Clin Neurosci 2016;18:447-57.

21. LeGates TA, Kvarta MD, Thompson SM. Sex differences in antidepressant efficacy. Neuropsychopharmacology 2019;44:140-54.

22. Benchimol EI, Smeeth L, Guttmann A, RECORD Working Committee, et al. The REporting of studies Conducted using Observational Routinely-collected health Data (RECORD) statement. PLoS Med 2015;12:e1001885.

23. Crossley KM, Callaghan MJ, van Linschoten R. Patellofemoral pain. BrJ Sports Med 2016;50:247-50.

24. Glaviano NR, Kew M, Hart JM, Saliba S. Demographic and epidemiological trends in patellofemoral pain. Int J Sports Phys Ther 2015;10:281-90.

25. Hauschild VD, Hauret K, Richardson M, Jones HB. A taxonomy of injuries for public health monitoring and reporting. Army Public Health Center. 2017. Available from: https://apps.dtic.mil/dtic/tr/ fulltext/u2/1039481.pdf.

26. Mihaylova B, Briggs A, O'Hagan A, Thompson SG. Review of statistical methods for analysing healthcare resources and costs. Health Econ 2011;20:897-916.

27. Trouvin A-P, Perrot S. New concepts of pain. Best Pract Res Clin Rheumatol 2019;33:101415.

28. Nekovarova T, Yamamotova A, Vales K, Stuchlik A, Fricova J, Rokyta R. Common mechanisms of pain and depression: are antidepressants also analgesics? Front Behav Neurosci 2014;8:99.

29. Clement S, Schauman O, Graham T, et al. What is the impact of mental health-related stigma on helpseeking? A systematic review of quantitative and qualitative studies. Psychol Med 2015;45:11-27.

30. Cheung RTH, Zhang Z, Ngai SPC. Different relationships between the level of patellofemoral pain and quality of life in professional and amateur athletes. PM R 2013;5:568-72.

31. Assa T, Elbaz A, Mor A, et al. Gait metric profile of 157 patients suffering from anterior knee pain. A controlled study. Knee 2013;20:40-4.

32. Domenech J, Sanchis-Alfonso V, López L, Espejo B. Influence of kinesiophobia and catastrophizing on pain and disability in anterior knee pain patients. Knee Surg Sports Traumatol Arthrosc 2013;21:1562-8.

33. Hayes JP, Vanelzakker MB, Shin LM. Emotion and cognition interactions in PTSD: a review of neurocognitive and neuroimaging studies. Front Integr Neurosci 2012;6:.

34. Wiech K, Shriver A. Cognition doesn't only modulate pain perception; it's a central component of it. AJOB Neurosci 2018;9:196-8.

35. Rhon DI, Lentz TA, George SZ. Utility of catastrophizing, body symptom diagram score and history of opioid use to predict future health care utilization after a primary care visit for musculoskeletal pain. Fam Pract 2020;37:81-90.

36. Quartana PJ, Campbell CM, Edwards RR. Pain catastrophizing: a critical review. Expert Rev Neurother 2009;9:745-58.

37. Sharifzadeh Y, Kao M-C, Sturgeon JA, Rico TJ, Mackey S, Darnall BD. Pain catastrophizing moderates relationships between pain intensity and opioid prescription: nonlinear sex differences revealed using a learning health system. Anesthesiology 2017;127:136-46.

38. Gensel JC, Donahue RR, Bailey WM, Taylor BK. Sexual dimorphism of pain control: analgesic effects of pioglitazone and azithromycin in chronic spinal cord injury. J Neurotrauma 2019;36:2372-6.

39. Weimer MB, Macey TA, Nicolaidis C, Dobscha SK, Duckart JP, Morasco BJ. Sex differences in the medical care of VA patients with chronic non-cancer pain. Pain Med 2013;14:1839-47.

40. McGraw K. Gender differences among military combatants: does social support, ostracism, and pain perception influence psychological health? Mil Med 2016;181:80-5.

41. Kazanis W, Pugh MJ, Tami C, et al. Opioid use patterns among active duty service members and civilians: 2006-2014. Mil Med 2018;183:e157-e164. 